\title{
A Tile-Based 3D Frame Using A Reconfigurable Display Matrix
}

\author{
Daqing Xue \\ Roger Crawfis \\ The Ohio State University \\ \{xue, crawfis\}@cis.ohio-state.edu
}

\section{Introduction}

We develop a tile-based 3D frame with a reconfigurable display matrix to reproduce the scene. Our display matrix can be configured into many scenes without building the exact geometric models as in Raskar et al.'s shader lamp [1]. By adjusting the tile positions, we can reproduce different scenes on the display matrix or add interactivity by varying the image depth buffer in real time.

\section{System Overview}

Figure 1 shows the diagram of our 3D frame system. The scene image is divided into the same number of the tiles as the display matrix, pre-warped and projected from the left projector onto the non-planar display surface of the matrix. The displacement of each tile is determined by the average depth of the pixels that project onto it. Since the display surface is a discontinuous manifold, shadows occur due to the illumination from the left projector and may be visible to the user in the walking area. The right projector is used to illuminate or hide these shadow regions. The user at the center of the walking area sees a geometrically correct scene image on the non-planar display wall. Users, viewing the display off center, see a geometric approximation to the 3D model with per-pixel illumination.

To change the display surface interactively, we have developed a robotic display matrix. Figure 2 shows our matrix with a $16 \times 8$ set of display tiles. Each tile is driven by an inexpensive servo motor. We can adjust the tiles' displacement to create different scenes as well as provide interactivity or dynamic scene support by modifying the tiles' displacement in real time.

\section{Texture Image Warping}

Each tile is mapped with its counterpart in the projected scene view with image or texture blocks of equal size initially. The display tile is then extruded according to the average depth of the pixels in their image tile. This changes the projected texture and thus the average depth. We iteratively adjust the tile's displacement, the mapped texture, and its corresponding depth until we have a good warping. Then all tiles on the display wall are rendered to the left and right projectors, respectively, and mapped with the proper texture tiles created from the above step. For the right projector, we use the tile geometry to calculate the projected shadows from the left projector. We use this shadow mask to restrict the energy projected from the right projector. Finally, these two warped images are projected from the left and right projectors, respectively, to reproduce the virtual environment on the scene-shaped display wall.

\section{Application}

We have applied our system to render a torus scene, a dynamic scene of a bouncing teapot, and some landscape scenes. Figure 3 shows a picture of the torus scene on the display matrix taken from the center in the walking area. The landscape scene and the bouncing teapot scene can be visited at http://www.cis.ohiostate.edu/ xue/research/siggraph03-sketch.

\section{References}

[ 1] R. Raskar, G. Welch, K. Low, and D. Bandyopadhyay (2001). "Shader Lamps: Animating Real Objects With Image-Based Illumination," Rendering Techniques 2001, Proceedings of the Eurographics Workshop in London, United Kingdom. S. J. Gortler and K. Myszkowski. University College London (UCL), London, England, Springer, NewYork: 89-102.

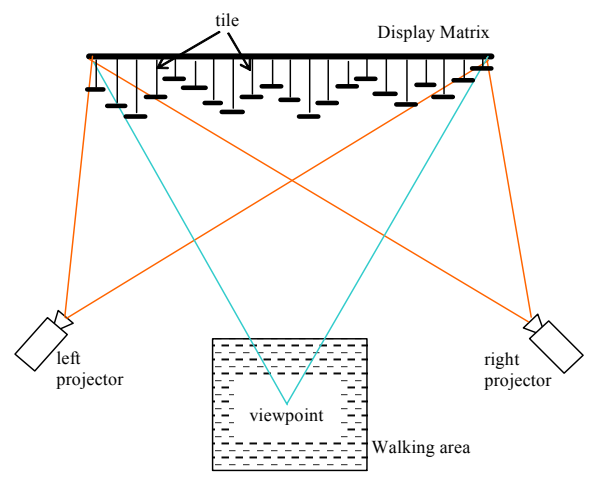

Figure 1: The top view of the tile-wise 3D frame.

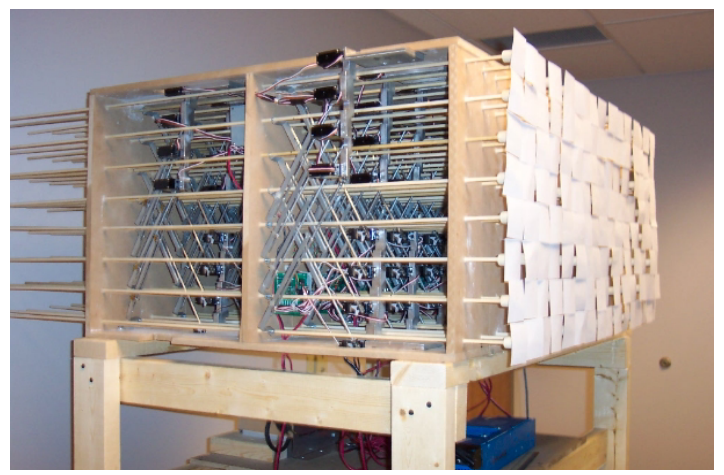

Figure 2: A 16×8 reconfigurable display matrix.

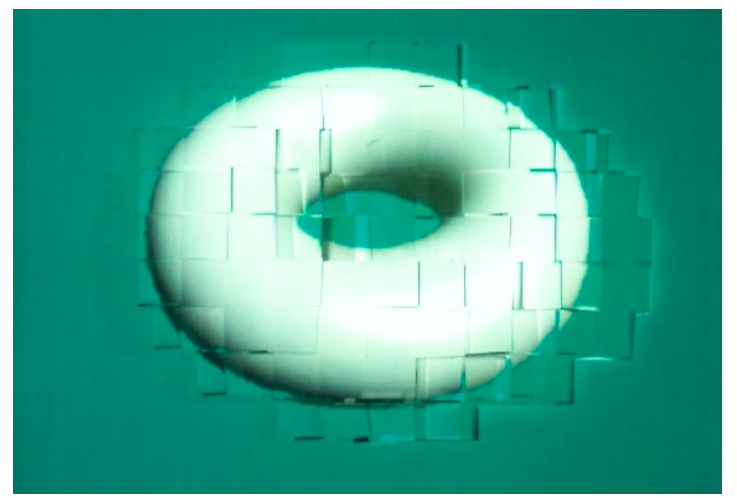

Figure 3: A torus scene image projecting onto the display matrix. 